\title{
The New Fontiers of Service Systems Engineering
}

\author{
Automation, Interaction, Openness and Learning
}

\author{
Tilo Böhmann · Jan Marco Leimeister • \\ Kathrin Möslein
}

Published online: 19 July 2018

(C) Springer Fachmedien Wiesbaden GmbH, part of Springer Nature 2018

Services are an abundant form of value creation in many countries. Even advanced products and technologies are increasingly made available as services, as exemplified by the dramatic expansion of cloud-based analytics and artificial intelligence. Digital transformation drives servitization. The process of digital transformation amplifies the trend to changing business models, towards offering and selling the utility of a product or a service - everything becomes a service, enabled though digital technologies. Traditional manufacturing sees advances of services through smart services and industry 4.0. Service industries themselves are also transforming into high tech ventures. Retail, finance, media, mobility, to name just a few industries, have become hotbeds of digital services that thrive on advances in information technology.

Advanced economies are thus characterized by a strong prevalence of complex service systems.

Prof. Dr. T. Böhmann

Department of Informatics, Research Group IT Management and Consulting (ITMC), Universität Hamburg, Vogt-Kölln-Str. 30, 22527 Hamburg, Germany

e-mail: tilo.boehmann@uni-hamburg.de

Prof. Dr. J. M. Leimeister $(\square)$

University of St. Gallen, St. Gallen, Switzerland

e-mail: JanMarco.Leimeister@unisg.ch

Prof. Dr. J. M. Leimeister

Universität Kassel, Kassel, Germany

Prof. Dr. K. Möslein

Universität Erlangen-Nürnberg, Nuremberg, Germany

e-mail: kathrin.moeslein@fau.de

Prof. Dr. K. Möslein

HHL Leipzig, Leipzig, Germany
Yet, despite all technological advances, there are critical limits to what technologies can do in services. Emphatic interaction, creative solutions, and authentic experiences all remain mostly the domain of human actors in service systems. Services thus require a balanced collaboration between human and machine actors. Combining the "best of both worlds" in a systematic way, so that they together achieve things none of them could achieve alone, is a promising future vision for service systems engineering.

This is why we proposed a systems perspective for the design and engineering of service systems, and service systems in our understanding are socio-technical systems. A systems view emphasizes the interplay of human and machine elements of service systems. When we proposed service systems engineering in 2014 (Böhmann et al. 2014) as a key area for information systems research we envisioned research needs in three areas: service architecture, service interactions, and resource mobilization. Digitalization has since then further changed the competitive landscape for services, thus inviting a fresh look at research priorities in this field. The digital transformation changes service systems even further, they become increasingly automated, interactive, open, and learning systems.

Automation more and more emphasizes the use of algorithms to take key decisions in service processes as well as the processing of transactions or activities without human interventions. In recent years, we have witnessed the successful design of automated service systems, e.g., in floating car sharing services in which users locate, open, and pay for car sharing just by interacting with a mobile app. Also, platform business models are generally exemplars of highly automated services. Automation is not any more an approach applied to previously created service systems but a critical design parameter for successful designs right from the start. Scalability of services is the 
reason for automation-by-design. Unless automated, services face unfavorable economics when scaling to a larger user population. The ubiquity of IT in everyday devices, comprehensive network coverage and abundant data are strong drivers for this development.

Interaction focuses on the novel forms of engaging actors in service systems through digital channels, binding users to the system and providing data-based individualized unique experiences. All value creation in services is value in context and value in use - focus on improving interactions helps increasing value creation in service systems.

We now witness conversational agents changing emerging and changing may areas, more and more transaction conducted through natural language assistants, such as Alexa, Google Assistant, or Siri. Augmented and virtual reality are becoming more and more a reality for service interactions. Yet, we have just scratched the surface for understanding the interplay between such digital channels and human interaction or for developing design knowledge for successful interaction models in service systems. Designing for "humans in the loop" in a way that creates superior experience and sustainable work processes is still an open challenge.

Open denotes that digital service systems are often ecosystems with boundaries in flux and open interfaces that allow for a more dynamic form of orchestrating services. Openness can refer to resources (openly accessible, e.g., through APIs, etc.), processes (primarily participatory processes, e.g., as through crowdsourcing, open innovation, open source) and as an outcome (e.g., "democratizing" effects as in open business, open education, open government, open science, etc.). Through open interfaces, services can be combined with and/or augmented by other services and external data, using this for creating even open resources. Managing architectures and boundaries of such open service systems and/or the decisions on which service systems to join become critical design decisions in service system engineering.

Finally, learning points to the use of human and machine learning for rapid evolution of service systems, enabling service systems to adapt and contextualize value co-creation processes. Recently, substantial changes in the way organizations develop and operate digital services are gaining momentum. Agile development methodologies as well as the tight integration of development and operations (DevOps) advocate fast learning and experimentation in order to understand user preferences by analyzing their interaction with real systems. In the language of design science research, naturalistic evaluation thus becomes the new normal as data on user behavior becomes ubiquitous. Similarly, machine learning creates the opportunity for service systems that evolve their knowledge and activity based on data.

Service systems engineering will thus need to evolve in response to these developments. This can take different forms. A key way to advance the knowledge base is the evolution of concepts, models, and methods for service systems engineering, facilitating the systematic design of digital service systems. In addition, the advancement of new technologies creates the opportunity to advance the design of algorithms or components for service systems that address common problems and have wide-ranging applications.

For business and information systems engineering scholars, working on design knowledge for successfully used service systems with humans in the loop, requires a socio-technical system perspective. We believe that the combination and integration of social, technological, economic and even societal design elements will become even more relevant in the future.

In this special issue, we present three papers that show advances along these lines. In the first paper the authors propose recombinant service systems engineering as a novel approach to service systems engineering. Recombinant innovation refers to reusing and integrating resources that were previously unconnected. We can assume that many service innovations will be based on adding, dissociating, and associating existing value propositions by accessing internal and external resources instead of designing them from scratch. Beverungen et al. illustrate their findings using a predictive maintenance case of agricultural machines.

In the second paper, Overhage et al. focus on Modular SaaS platforms with software services, micro services, and open API structures providing new opportunities to realize even highly customized solutions in the cloud. They present the Business-Oriented Service Description Language (BoSDL) in order to facilitate the assessment of services against functional requirements enabling customers to discriminate between offered services and choose those best fulfilling the requirements. It consists of a meta-model with rules to describe the business logic, that is, the functionality of a software service from a business-oriented perspective; a textual presentation format based on English natural language; and a graphical notation based on the UML. The approach is evaluated in controlled experiments, indicating its favorability compared to other existing approaches.

In the third paper, Bortlik et al. address a service provider selection problem in contextualized and collaborative service systems. They develop a heuristic technique applying a decomposition of the users' global constraints and a local service selection. They aim to determine a feasible service composition for each participating user 
while taking the users' individual preferences and constraints as well as context information into account. The evaluation of the heuristic technique uses a real-world scenario from the tourism domain to illustrate the heuristics' potential advantages for decision makers in multi user context-aware service systems.

We hope you find the special issue insightful and worthwhile and we invite you to join our efforts in developing useful and relevant design knowledge in and for service systems engineering.

\section{References}

Böhmann T, Leimeister JM, Möslein K. (2014) Service Systems Engineering - A Field for Future Information Systems Research. Bus Inf Syst Eng 6(2):73-79 